\title{
Modeling and robust control algorithms for a linear belt driven system
}

https://doi.org/10.1515/comp-2018-0010

Received October 13, 2017; accepted June 13, 2018

\begin{abstract}
This paper proposes the mathematical modeling and robust control algorithms for linear belt system with the help of sliding mode control approach. Due to the elasticity of the belt, the presence of frictions, and the un-modeled dynamics, conventional controllers cannot provide precise position control of carriage. Dealing with this kind of system, a robust controller is needed and the chattering-free sliding mode control (SMC) approach is used to design the robust controller. A belt stretching estimator is also incorporated into the control law. Simulations show that the system is free from chattering and robust to disturbances. The reference tracking position is performed with the minimal errors to an extent that can be considered negligible. The time for reaching the reference tracking position is very fast. The system is safe for all mechanical and electrical devices.
\end{abstract}

Keywords: robust controller, linear belt, sliding mode control, chattering free, un-modeled dynamics, friction model, equivalent control

\section{Introduction}

Usage of belt drives has gathered significant attention and become the most common method of power transmission used in most common systems/devices. Another significant sphere where the belt drives are exhaustively used includes industrial transportation systems where they are often called conveyor belts. Belt drives are better and more popular than other power transmission mechanisms such as the chains or the gears because they have a number of advantages including low cost, simple, low maintenance,

\footnotetext{
*Corresponding Author: Vu Trieu Minh: Department of Electrical Power Engineering and Mechatronics Tallinn University of Technology, 19086 Tallinn, Estonia, E-mail: trieu.vu@ttu.ee

Mart Tamre, Even Sekhri: Department of Electrical Power Engineering and Mechatronics Tallinn University of Technology, 19086 Tallinn, Estonia, E-mail: mart.tamre@ttu.ee, even.sekhri@ttu.ee
}

smooth operation, fast and flexible installation, noisefree, cleanliness, no lubrication needed, can absorb sudden shocks, wide speed ratio range operations, easy to visualize the failure, etc. in [1].

Presently, toothed belts (also called as timing belts) are a leading edge in industrial applications for power transmission with precise position tracking and rapidly changing dynamics (such as in 3D printers). The main advantages of toothed belts are high efficiency, long travel length, suitability to high speed applications and low cost. However, the main disadvantages of the belt drives are high tracking errors, uncertain dynamics and vibrations. The motion equations and the mathematic modellings of such a linear belt driven system is discussed in [2].

The controllers for belt drives are considered like PIDs by re-designing to cope-up higher-order-node in [3]. However, these controllers become unstable with high resonant frequencies. Further, they are not sufficient enough to deal with the tracking errors. Some additional methods such as signal processing and noise filtering are used to improve the belt drive performances in [4], but they are not sufficient for mechanical vibrations, plant variations, load-torque disturbances and uncertain dynamics. A number of other approaches like linear quadratic regulator (LQR), composite non-linear feedback (CNF), adaptive control and sliding mode control (SMC) have provided better control performances.

Every controller has its advantages and disadvantages. SMC has also a major drawback of discontinuous switching action so as to keep the system stable and to operate in accordance to the desired expectations. This means that the control must switch with infinite frequency to provide the total rejection to the uncertainties present in the system. This discontinuous mode leads to a phenomenon called "chattering" in [5], which is dangerous for mechanical systems and may lead to wear and tear of the parts involved and in extreme cases may even lead to the failure of the whole system.

In this research, a chattering-free SMC is considered to control the belt drive. Belt- stretch and its control are important to achieve the vibration-free performance in [6]. Thus, paramount importance is given to the belt-stretch 
in the present research endeavor. The control law is derived by the combination of the Lyapunov function and the SMC theory. The use of Lyapunov function allows stabilizing the system upon any uncertainties and disturbances. Therefore, the Lyapunov applications in [7] and in [8] are used to design the SMC.

Owing to its simplicity and robustness, SMC approach has attracted significant attention from researchers since 1980s. The achievements include almost all kinds of systems such as the SMC for the non-linear systems in [9], SMC for discrete systems in [10], SMC for time-delay systems in [11], and SMC for large systems in [12]. To address the problems of chattering and unknown bound of the uncertainties present, various methods such as the higher order SMC in [13], the adaptive SMC in [14], and the adaptive fuzzy SMC which combine the advantages of both SMC and fuzzy logic controllers are discussed in [15] and in [16].

The objective of this research is to design a robust controller for a linear belt driven system based on SMC to control the position and velocity of a carriage on with the ship model tagged. This is from a real project in Kuressaare, Estonia, to build a water cannel to test the ship navigational and hydrodynamic properties, such as the ship behavior/friction in the waves. The carriage is driven by two toothed belts and two motors as seen in Figure 1. The motive is to make the system robust enough to deal with the unknown parameters and disturbances whilst keeping the tracking position and velocity errors as low as possible. Advanced robust, adaptive control designs are referred to in [17], [18] and [19].

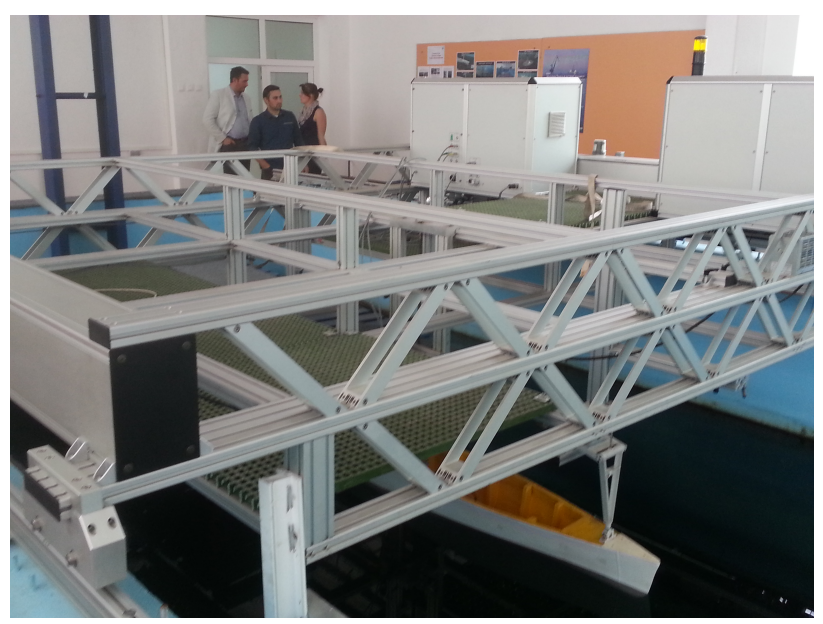

Figure 1: Real linear belt driven system in Kurresaare, Estonia.

The contents of this paper are as follows: Section 2 develops the mathematical model formulation of the system; Section 3 presents the SMC controller design; Section
4 presents the results of simulation; Section 5 draws the conclusions and recommendations.

\section{Mathematical model}

The considered system consists of a DC motor which provides the main motive force to the system, two pulleys with same dimensions that stretch the belt where the one which is connected to the motor and is the driving pulley, and the other is the driven pulley; a carriage (which is considered as the load-side of the system) whose tracking position and velocity errors are to be minimized ultimately; and the belt for force transmission from the driven pulley to the load carriage. The arrangement represents a complexcum-nonlinear distributed parameter system.

The following assumptions are made for the system: gear reduction ratio has its value equal to unity in the simulated system; the motor has negligible delay in providing high-dynamic torque response; the system is free from backlash of belt drives due to pre-tensioning; the link between motor shaft and belt drive's driving pulley is rigid; the belt elasticity is equivalent to a mass-less spring; the unknown disturbances/noises consist of friction present between DC motor and the driving pulley and carriage guiding rail transmission guiding channels. Figure 2 shows the diagrammatic representation of the equivalent spring-mass system in lieu of these underlying assumptions.

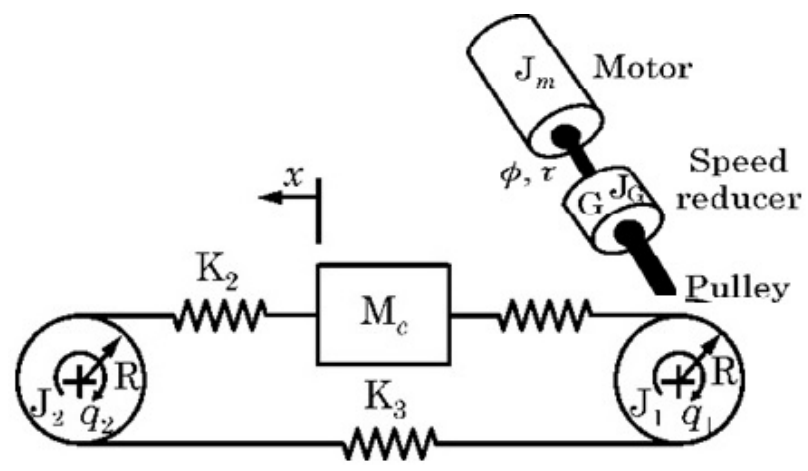

Figure 2: Equivalent spring-mass system.

Using modal analysis, the sixth-order dynamic equations for the system are depicted in equations (1), (2), and (3) in [2]. For generating these equations the detailed anal- 
ysis is made in [6].

$$
\begin{aligned}
& \left(J_{1}+G^{2}\left(J_{G}+J_{m}\right)\right) \ddot{q}_{1}+\tau_{f 1}=G\left(-R\left[K_{1}(x)\left(R q_{1}-x\right)\right.\right. \\
& \left.-K_{3}\left(R q_{2}-R q_{1}\right)\right], \\
& J_{2 \ddot{q}_{2}}+\tau_{f 2}=R\left[K_{2}(x)\left(x-R q_{2}\right)-K_{3}\left(R q_{2}-R q_{1}\right)\right], \\
& M_{c} \ddot{x}+f_{f}=K_{1}(x)\left(R q_{1}-x\right)-K_{2}(x)\left(x-R q_{2}\right),
\end{aligned}
$$

where, $J_{G}, J_{m}$ - Moment of Inertia of the speed reducer and the motor, respectively; $J_{1}, J_{2}-$ Moment of Inertia of driving and the driven pulley, respectively; $M_{c}$ - Carriage mass; $G$ - Speed reducer gear ratio; $R$ - Radius of the pulleys; $K_{1}, K_{2}, K_{3}$ - Position dependent belt elasticity coefficients; $q_{1}, q_{2}, \phi$ - Angular position of the driving pulley, driven pulley, and the motor, respectively; $x$ - Position of carriage; $\tau$ - Torque developed by the motor; $\tau_{f 1}, \tau_{f 2}-$ Friction torque which affects the pulleys; $f_{f}$ - Friction force on the carriage.

The $6^{\text {th }}$ order model discussed above is a three-mass model and it is a highly-coupled and non-linear system with external disturbances which enter both in the drivingside, i.e., motor-side and also on the load-side of the system. An important point to be considered is that the inertias at the load-side and the motor-side are high as compared to those at the driving and driven pulleys [6]. So, the $6^{\text {th }}$ order model is reduced to $4^{\text {th }}$ order model (two-mass model) as described in equations (4), (5), and (6). The twomass model includes only the first resonance.

$$
\begin{gathered}
J \ddot{\varphi}+\tau_{f}=\tau-L K w, \\
M \ddot{x}+f_{f}=K w, \\
w=L \varphi-\chi,
\end{gathered}
$$

where: $J$ - Motor inertia; $M$ - Mass on the load side (approx. to the Cart Mass); $\tau_{f}$-Motor Side friction torque that perturb the system ; $f_{f}$ - Force of friction in the system; $K$ - Coefficient of Elasticity of the belt; $w$ - Belt-stretch; $L$ Transmission constant of the linear belt-drive $=R / G$;

Belt-stretch is an important consideration. The model can hence be modified according to the vibration analysis. It is to be noted that to simplify the calculations, the parameter " $L$ ", i.e., transmission coefficient is assumed to be unity while finding the new set of equations for the modified model. The modified model can be written as:

$$
\begin{gathered}
J \ddot{w}+K_{w} w=\tau-\tau_{w f}, \\
M \ddot{x}+f_{f}=K,
\end{gathered}
$$

where: $\tau_{w f}=\tau_{f}-\kappa f_{f}, K_{w}=K(1+\kappa), \kappa=J / M$ (inertia ratio).

The resonance frequency of the elastic belt-drive system is given by Equation (9):

$$
\omega_{0}=\sqrt{\frac{K}{J}(1+\kappa)}
$$

The control model for the modified system where beltstretch is considered is shown in Figure 3. The system has a control input signal $\tau$. It has two parts where the first one is for the Belt-Stretch Dynamics and the second one is for the Load Side Dynamics. Both these parts are described by their independent nominal and linear $2^{\text {nd }}$ order dynamics. The disturbance torque perturbs the belt-stretch side and the friction force perturbs the load side.

In real life, the main problems in such a system arise from many factors some of which include: Elasticity of the belt; Load-position dependent friction; Non-linearity of induced belt forces; Large friction effects; etc. The prime interest is to generate the control scheme for the system mentioned in Figure 3 so that the position tracking can be simulated and the position error of the cart position can be minimized. Also, from the simulation, results of various other parameters can also be fetched.

\section{Free chattering SMC design}

The design of SMC is two-stepped process: Defining the switching hyperplane or switching surface or sliding surface; and Designing the control law. First, the switching hyperplane often designated by " $\sigma$ " is defined which is basically dependent on the desired dynamical behavior of the system. This $\sigma$ actually provides a measure of the distance of the state-trajectory from the sliding surface located at $\sigma=0$. Secondly, a control law is designed to drive the states onto the pre-defined sliding surface. Also, it makes sure that they remain there and slide along the sliding surface or in the bounded vicinity of that sliding surface. The control is continuous in all the segmented regions but it alters the structure when the dynamics of the state-trajectory crosses the boundary defined by the sliding surface, i.e., sliding manifold.

The ultimate goal is to find the control input which takes the system towards the sliding surface manifold and then restricts it there despite of the perturbations present in the system which basically include all the uncertainties of the system.

Let a Single Input-Single Output (SISO) non-linear considered mechanical system in the state-space be defined as:

$$
\dot{z}_{i}=z_{i+1} \text {, }
$$




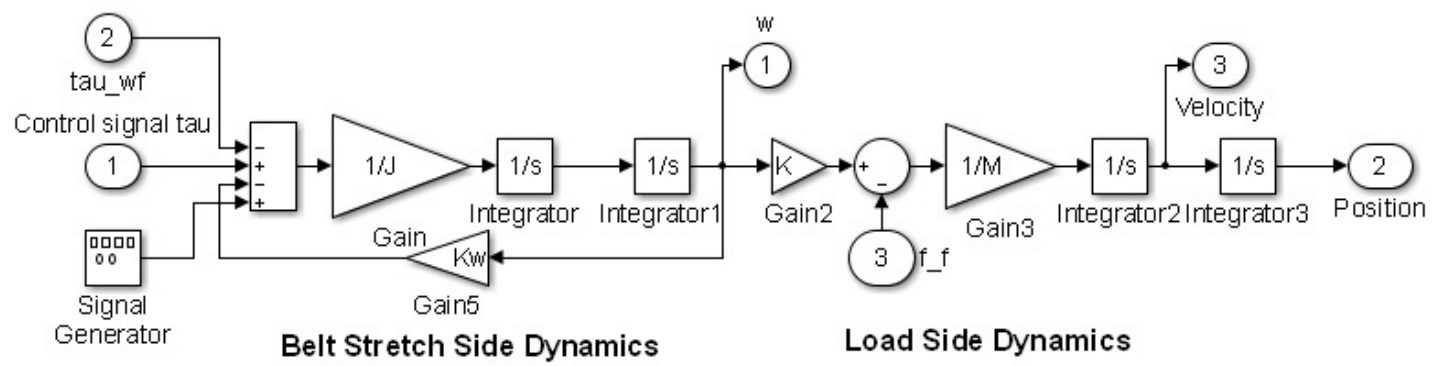

Figure 3: Simulink scheme of linear belt-driven system.

$$
\dot{z}_{n}=f(z)+b(z) u+d(t)
$$

where: $i=1, \ldots, n-1 ; z^{T}=\left[z_{1} \ldots, z_{n}\right]$ is a system state vector; $u$-scalar input; $f(z)$ - the bounded non-linear driving term of system-state vector; $b(z)$ - the bounded non-linear control gain of the system-state vector; $d(t)$ - the bounded scalar disturbances.

Now, the prime goal is to determine control signal " $u$ " that restricts the motion of the system-states $z$ to the predefined sliding surface $\sigma(z, t)=0$ even in the presence of $f(z), b(z)$ and $d(t)$. The convergence (of system states) to the sliding surface is called the reaching phase. The motion within the sliding manifold is called the sliding phase.

The control with discontinuities on sliding manifold is working on the following defined principles (termed as switching function):

$$
u= \begin{cases}u^{+}, & \sigma(z, t)>0, \\ u^{-}, & \sigma(z, t)<0 .\end{cases}
$$

Here, $u^{+}$and $u^{-}$are selected such that the Lyapunov function candidate has its derivative to be negativedefinite. But, the discontinuous control in many applications leads to failure of VSS which further leads to "chattering" which is an important issue while dealing with the system under consideration. Hence, the chattering-free SMC is to be used in this task. For this purpose, another additional state of the system is needed as introduced in [7] and also discussed in [9] to eliminate the discontinuities on the control signal. So, it yields,

$$
\begin{gathered}
\dot{z}_{i}=z_{i+1}, \\
z_{n+1}=g(z, u)+b(z) \dot{u}+\dot{d}(t),
\end{gathered}
$$

where $g(z, u)=\dot{f}(z)+\dot{b}(z) u, i=1, \ldots, n$.

The condition which is to be fulfilled so that the system states start moving towards; and finally reach the sliding surface is called the reaching condition. The solution given by $\sigma=0$ will have at least asymptotic stability if the control satisfies the condition that the derivate of any assumed Lyapunov's candidate function will be semi-negative definite. To meet this criterion, considering the Lyapunov candidate of the form:

$$
V(\sigma)=\sigma^{2} / 2 .
$$

The squared distance to the surface (as measured with term $\sigma^{2}$ ) decreases along the system trajectory. Hence, it will constrain the trajectories to actually point towards the pre-defined sliding surface. Once the system reaches the surface, the system trajectories will then be on the surface.

The reachability condition can be proved when the condition $\sigma, \dot{\sigma}<0$ is fulfilled. The robust law is chosen with proportional rate given by $\dot{\sigma}=-D \sigma, D>0$ where $D$ is the damping coefficient arbitrarily chosen to achieve a required rate of robustness of the closed-loop system. The value of $D$ determines the disturbance rejection and ideally the value of gain $D$ should be as high as possible. But, it is not possible in the practical applications. The value of $D$ is limited by various factors including: un-modeled dynamics of electro-magnetic torque; noise levels; discrete control algorithm implementation.

The derivative of Lyapunov's candidate function $V$ as stated by Equation (15) will thus have the form:

$$
\dot{V}=-D \sigma^{2}, D>0 \text {. }
$$

Now, from the condition $\dot{V}=\sigma \dot{\sigma}=-D \sigma^{2}$ and by application of the knowledge regarding the equivalent control method in which the equivalent control signal $u_{e q}$ is not the control action applied to the nominal plant. It can be imagined as representing (at an average) the same effect as shown by the applied discontinuous control and can hold the system on the sliding surface:

$$
\dot{u}=\dot{u}_{e q}+D \sigma .
$$


The obtained equivalent control $u_{e q}$ is actually the solution of $\left.\dot{\sigma}\right|_{\sigma=0}=0$.

The control law $u$ can be obtained as:

$$
u=\int_{0}^{t} \dot{u} d t .
$$

This control assures invariant motion of the system in the sliding mode if the value of the disturbance $d(t)$ complies to matching conditions. The control will be achieved when the derivative of Lyapunov candidate is negative, i.e.,

$$
\dot{V}=\sigma \dot{\sigma}=-D \sigma^{2}<0 .
$$

The task of designing the sliding surface or sliding manifold is an important step which is constructed in a system state-space. Thus, $\sigma$ combines system's statevariables in such a way that the system's motion in the sliding manifold is asymptotically stable. The definition of the system states is in accordance to the adopted system model. In case of higher-order sliding mode approach, the original system states can be extended so as to achieve the smooth control.

For the system considered, the conventional SMC design approach involves switching function of first order which could be taken as:

$$
\sigma=\Delta \dot{x}-c \Delta x
$$

where: $\Delta x-$ the error signal $=r(t)-x$, i.e., reference position - actual position; $c$ - our design variable.

The SMC which is free from chattering for the linear belt-driven system must have a switching function of second-order in the motion control. Hence, taking the switching function to be:

$$
\sigma=\Delta \ddot{x}+K_{v} \Delta \dot{x}+K_{p} \Delta x,
$$

where: $\Delta x$ - corrected position; $\Delta \dot{x}$ - corrected velocity; $\Delta \ddot{x}$ - corrected acceleration; $K_{v}$ and $K_{p}$ - positive control gain values to shape the second-order dynamic behavior of the error in desired position.

But for elastic systems, the switching function is given by:

$$
\sigma=\Delta \ddot{x}+K_{v} \Delta \dot{x}+K_{p} \Delta x+\gamma(\ddot{w}+\alpha \dot{w}),
$$

where $\alpha$ and $\gamma$ - arbitrarily chosen positive control gains in order to reduce the vibrations due to belt compliance and elasticity so as to shape asymptotically stable motion dynamics on the sliding manifold.

The portion of belt-stretch dynamics is added to switching function definition $(\ddot{w}+\alpha \dot{w})$. It basically aims at coping with resonant frequency $\omega_{0}$ and also to achieve asymptotically stable dynamics of motion.
A little consideration to equation (22) says that if the belt is stiff then both $\ddot{w}$ and $\dot{w}=0$ and we will reach equation (21).

For the controller implementation, the only signals we require are position and the velocity of the system. The other required model parameters are the equivalent mass, i.e., $J+M$, the moment of inertia of the motor, i.e., $J$ and lastly the resonance frequency, i.e., $\omega_{0}$ of the system. And then the other control parameters, i.e., $K_{v}, K_{p}, \alpha, \gamma$ are designed.

The Simulink description of the belt-stretch consideration being implemented and the calculation of the control law for the system is as shown in Figure 4.

The constructed sliding manifold as described in Equation (22) allows the steady state position error to reach the zero value assuring the operation to be vibrationfree. In order to give a little more explanation about choice of the switching function, $\sigma$ has been opted such that it complies with the order of the system. Also, it involves position tracking error and its order up to the $2^{\text {nd }}$ order along with the dynamics of vibration suppression, i.e., $\dot{w}$ and $\ddot{w}$.

It is to be noted here that higher order derivatives of position error are simply not available in the practice. All the variables involved in $\sigma$ are then available in the implemented control law which actually requires the integral of $\sigma$.

Considering control engineer's perspective, $\sigma$ is a measure for a distance to sliding manifold. The sliding manifold is constructed so as to bring the driving position error to zero while operating at vibration-free mode. In practice, the ultimate convergence is hard to guarantee, especially, in the case of simplified version of the control law. However, by the high-gain approach, it can asymptotically converge to a vicinity of the sliding manifold at a fair robustness.

In practical scenarios, it is difficult to observe beltstretch's first and second order derivatives. So, Equation (8) belt-stretch is computed as:

$$
w=\frac{M}{K} \ddot{x}+\frac{1}{K} f_{f} .
$$

The first and second order derivatives are hence calculated by differentiating " $w$ ". In MATLAB Simulation, it can be done by using the "Derivative" blocks from Simulink library (in Figure 4).

Now, as discussed earlier that the control law needs the derivative of switching function $\sigma$. So, differentiating Equation (22),

$$
\dot{\sigma}=\Delta \dddot{x}+K_{v} \Delta \ddot{x}+K_{p} \Delta \dot{x}+\gamma(\dddot{w}+\alpha \ddot{w}) .
$$




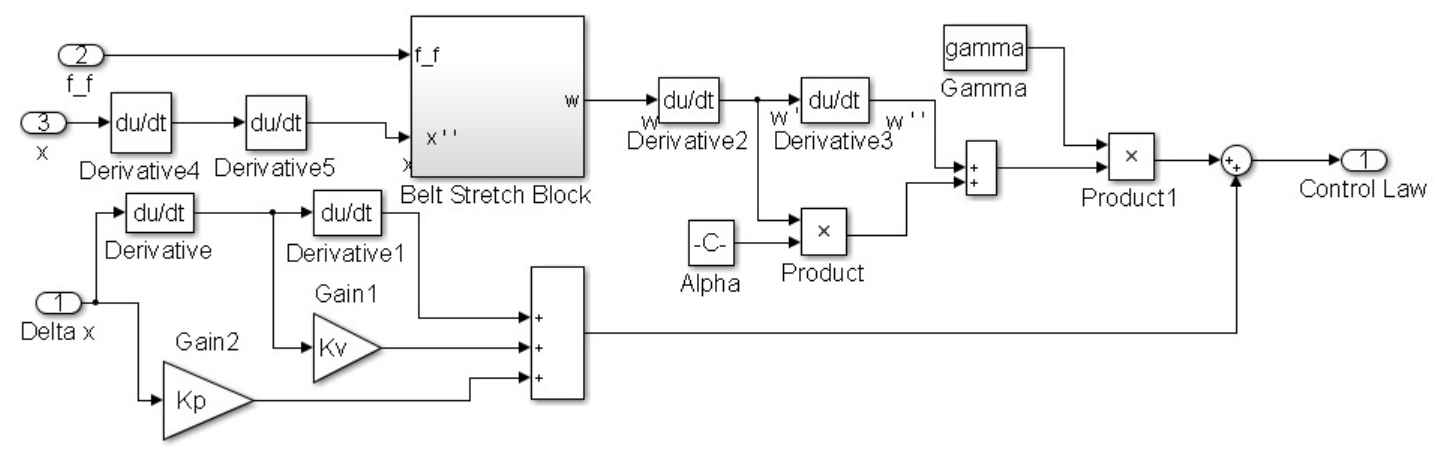

Figure 4: Control law including the belt-stretch.

Here, $\ddot{w}$ is obtained by rearranging the terms involved in Equation (7) as:

$$
\ddot{w}=-\omega_{0}^{2} w+\frac{\tau-\tau_{w f}}{J} .
$$

$\dddot{w}$ is calculated by differentiating the Equation (25), i.e., calculating $d \ddot{w} / d t$.

So,

$$
\dot{\sigma}=\Delta \dddot{x}+K_{v} \Delta \ddot{x}+K_{p} \Delta \dot{x}+\gamma\left(-\omega_{0}^{2} \dot{w}+\frac{\dot{\tau}-\tau_{w f}}{J}+\alpha \ddot{w}\right) .
$$

Now, from condition $\dot{\sigma}=0$ where $\dot{\tau}$ becomes $\dot{\tau}_{\text {eq }}$ :

$$
0=\Delta \dddot{x}+K_{v} \Delta \ddot{x}+K_{p} \Delta \dot{x}+\gamma\left(-\omega_{0}^{2} \dot{w}+\frac{\dot{\tau}_{e q}-\tau_{w f}}{J}+\alpha \ddot{w}\right) .
$$

Re-arranging the terms leads to:

$$
\gamma \dot{\tau}_{e q}=-J\left[\Delta \dddot{x}+K_{v} \Delta \ddot{x}+K_{p} \Delta \dot{x}\right]+J \gamma \omega_{0}^{2} \dot{w}-J \gamma \alpha \ddot{w}+\gamma \dot{\tau}_{w f} .
$$

Further rearrangement of terms yields:

$$
\dot{\tau}_{e q}=-\frac{J}{\gamma}\left[\Delta \dddot{x}+K_{v} \Delta \ddot{x}+K_{p} \Delta \dot{x}\right]+J\left[\omega_{0}^{2} \dot{w}-\alpha \ddot{w}\right]+\dot{\tau}_{w f} .
$$

Using the knowledge of resonance frequency (from Equation 29) we can deduce that,

$$
\omega_{0}^{2}=\frac{K}{J} \frac{(M+J)}{M} .
$$

Rearranging the terms in Equation 21 above gives the following:

$$
\frac{M}{K}=\frac{(M+J)}{J} \cdot \frac{1}{\omega_{0}^{2}} .
$$

Multiplying and dividing the equation of $\dot{\tau}_{e q}$ by $\frac{K}{M}$ we get:

$$
\dot{\tau}_{e q}=-\frac{M}{K} J \beta\left[\Delta \dddot{x}+K_{v} \Delta \ddot{x}+K_{p} \Delta \dot{x}\right]+J\left[\omega_{0}^{2} \dot{w}-\alpha \ddot{w}\right]+\tau_{w f}
$$

where $\beta=K / M \gamma=A$ gain value.

High value of $\beta$ can extend the robust bandwidth of the operation but it is always limited in the real life. For example, in belt-drives, it is normal to have force-transmission delay which may lead to unstable belt response. Hence, $\beta$ value is actually a result of design compromise. Also, $\beta=$ $\gamma^{-1} K / M>0$ is the design parameter which shapes the dynamics of the system motion when $\sigma=0$.

Substituting $\frac{M}{K}$ from Equation (31) to Equation (32):

$\dot{\tau}_{e q}=-\frac{\beta}{\omega_{0}^{2}}(M+J)\left[\Delta \dddot{x}+K_{v} \Delta \ddot{x}+K_{p} \Delta \dot{x}\right]+J\left[\omega_{0}^{2} \dot{w}-\alpha \ddot{w}\right]+\tau_{w f}$.

Now, equivalent control torque signal can be obtained by integrating the $\dot{\tau}_{e q}$ over the time limit 0 to $t$, i.e.

$$
\tau_{e q}=\int_{0}^{t} \dot{\tau}_{e q} d t .
$$

So, integrating Equation (33),

$\tau_{e q}=-\frac{\beta}{\omega_{0}^{2}}(M+J)\left[\Delta \ddot{x}+K_{v} \Delta \dot{x}+K_{p} \Delta x\right]+J\left[\omega_{0}^{2} w-\alpha \dot{w}\right]+\tau_{w f}$.

Here, in Equation (35), we can see that we need acceleration signal which is generally not available. We have only position and velocity sensors installed with-in the system.

So, to find $\ddot{x}$ we have to use Equation 28 where rearranging the terms provides:

$$
\Delta \ddot{x}=\frac{K w-f_{f}}{M} .
$$

Hence,

$$
\begin{aligned}
\tau_{e q}= & -\frac{\beta}{\omega_{0}^{2}}(M+J)\left[K_{v} \Delta \dot{x}+K_{p} \Delta x\right]-\frac{\beta}{\omega_{0}^{2}}(M+J)\left(\frac{K w-f_{f}}{M}\right) \\
& +J\left[\omega_{0}^{2} w-\alpha \dot{w}\right]+\tau_{w f},
\end{aligned}
$$




$$
\begin{aligned}
\tau_{e q}= & -\frac{\beta}{\omega_{0}^{2}}(M+J)\left[K_{v} \Delta \dot{x}+K_{p} \Delta x\right]-\frac{\beta}{\omega_{0}^{2}}\left(K w-f_{f}\right) \\
& +\frac{\beta}{\omega_{0}^{2}} \frac{J}{M}\left(K w-f_{f}\right)+J\left[\omega_{0}^{2} w-\alpha \dot{w}\right]+\tau_{w f}, \\
\tau_{e q}= & -\frac{\beta}{\omega_{0}^{2}}(M+J)\left[K_{v} \Delta \dot{x}+K_{p} \Delta x\right]-\frac{\beta}{\omega_{0}^{2}} K w+\frac{\beta}{\omega_{0}^{2}} f_{f} \\
& -\frac{\beta}{\omega_{0}^{2}} \frac{J}{M} K w+\frac{\beta}{\omega_{0}^{2}} \frac{J}{M} f_{f}+J\left[\omega_{0}^{2} w-\alpha \dot{w}\right]+\tau_{w f} .
\end{aligned}
$$

Now, substituting the value of $\tau_{w f}$ in Equation (39) we get:

$$
\begin{aligned}
\tau_{e q}= & -\frac{\beta}{\omega_{0}^{2}}(M+J)\left[K_{v} \Delta \dot{x}+K_{p} \Delta x\right]-\frac{\beta}{\omega_{0}^{2}} K w+\frac{\beta}{\omega_{0}^{2}} f_{f} \\
& -\frac{\beta}{\omega_{0}^{2}} \frac{J}{M} K w+\frac{\beta}{\omega_{0}^{2}} \frac{J}{M} f_{f}+J\left[\omega_{0}^{2} w-\alpha \dot{w}\right]+\tau_{f}-\kappa f_{f} .
\end{aligned}
$$

After re-arranging the terms we get:

$$
\begin{aligned}
\tau_{e q}= & -\frac{\beta}{\omega_{0}^{2}}(M+J)\left[K_{v} \Delta \dot{x}+K_{p} \Delta x\right]-\frac{\beta}{\omega_{0}^{2}} K w-\frac{\beta}{\omega_{0}^{2}} \frac{J}{M} K w \\
& +J \omega_{0}^{2} w-J \alpha \dot{w}+\tau_{f}+\left[\frac{\beta}{\omega_{0}^{2}}+\frac{\beta}{\omega_{0}^{2}} \kappa-\kappa\right] f_{f}, \\
\tau_{e q}= & -\frac{\beta}{\omega_{0}^{2}}(M+J)\left[K_{v} \Delta \dot{x}+K_{p} \Delta x\right] \\
& -J\left[\alpha \dot{w}+\left(\frac{\beta}{\omega_{0}^{2}} \frac{K}{J}+\frac{\beta}{\omega_{0}^{2}} \frac{K}{M}-\omega_{0}^{2}\right) w\right]+\tau_{f}+\xi f_{f},
\end{aligned}
$$

where $\xi=\kappa\left(\frac{\beta}{\omega_{0}^{2}}-1\right)+\frac{\beta}{\omega_{0}^{2}}$.

Solving the Equation (42) and substituting $\omega_{0}^{2}=$ $\frac{K}{J}\left[\frac{M+J}{M}\right]$ we get:

$\tau_{e q}=-\frac{\beta}{\omega_{0}^{2}}(M+J)\left[K_{v} \Delta \dot{x}+K_{p} \Delta x\right]-J\left[\alpha \dot{w}+\left(\beta-\omega_{0}^{2}\right) w\right]+\tau^{d i s t}$,

where $\tau^{\text {dist }}=\tau_{f}+\xi f_{f}$ - the system disturbance signal.

It is to be noted that $\xi$ is related to both the plantmodel parameters and enforced belt-stretch dynamics parameters.

In order to obtain a continuous control of the signal $\sigma$, the control law applies the condition $\dot{\sigma}=-D \sigma$ in order to obtain control signal of the form $u=u_{e q}+D \sigma$ as explained earlier.

But we do not have the perfect knowledge about the whole system and in reality, $\tau^{\text {dist }}$ is not a measurable entity so, $\tau_{e q}$ is replaced with the estimated value $\hat{\tau}_{e q}$. The estimate $\hat{\tau}_{e q}$ does not assure the convergence to the sliding surface hence the discontinuous term is also added to it as described in Equation (44).

$$
\tau=\hat{\tau}_{e q}+\frac{\beta}{\omega_{0}^{2}}(J+M) D \sigma .
$$

Performance of robust controller as obtained from Equation (44) plays an important role in desensitizing the system from the disturbances. This also allows for implementation of the rapid vibration-free belt response which can further aid in rejecting the load side equation.

Equivalent control estimation is determined by:

$$
\hat{\tau}_{e q}=\frac{\beta}{\omega_{0}^{2}}(J+M) a^{c}-J\left(\alpha \dot{\omega}+\left(\beta-\omega_{0}^{2}\right) w\right),
$$

where $\mathrm{a}^{c}=K_{v} \Delta \dot{x}+K_{p} \Delta x$.

Now, the control torque signal is given by Equation (46) as:

$$
\tau=\int_{0}^{t} \dot{\tau} d t=\hat{\tau}_{e q}+\int_{0}^{t} \dot{\tau}_{S M C} d t .
$$

The Equation (46) has 2 components: estimation of equivalent control; and estimation of disturbance and convergence to pre-defined sliding manifold.

Using Equations (44) to (46), the system motion projection on $\sigma$-space is governed by:

$$
\dot{\sigma}+D \sigma=\frac{\omega_{0}^{2}}{\beta} \frac{\dot{\tau}^{d i s t}}{J+M} .
$$

Equation (47) in combination with the condition $\sigma \dot{\sigma}=-D \sigma^{2} ; D>0$, proves the system's asymptotically stable reaching phase. The convergence is dictated by the right side of Equation (47).

Having a stable solution where $\sigma=0$ can be guaranteed if $\dot{\tau}^{\text {dist }}=0$, i.e., it should be constant. Also, then the derivative of considered Lyapunov's function candidate is negative definite, i.e.,

$$
\dot{V}=-D \sigma^{2}, \quad D>0 .
$$

In systems with fast sampling rate, the fast convergence rate can be achieved. If the rate of change of disturbance is low, i.e., $\dot{\tau}^{\text {dist }}=0$ then the control law will keep the system states in the vicinity of the pre-defined sliding manifold.

\section{Simulation results}

The system considered in the research being simulated in MATLAB-Simulink is depicted in Figure 5.

The control and simulation system as shown in Figure 5 consisted of four main blocks: The nominal plant (I) 


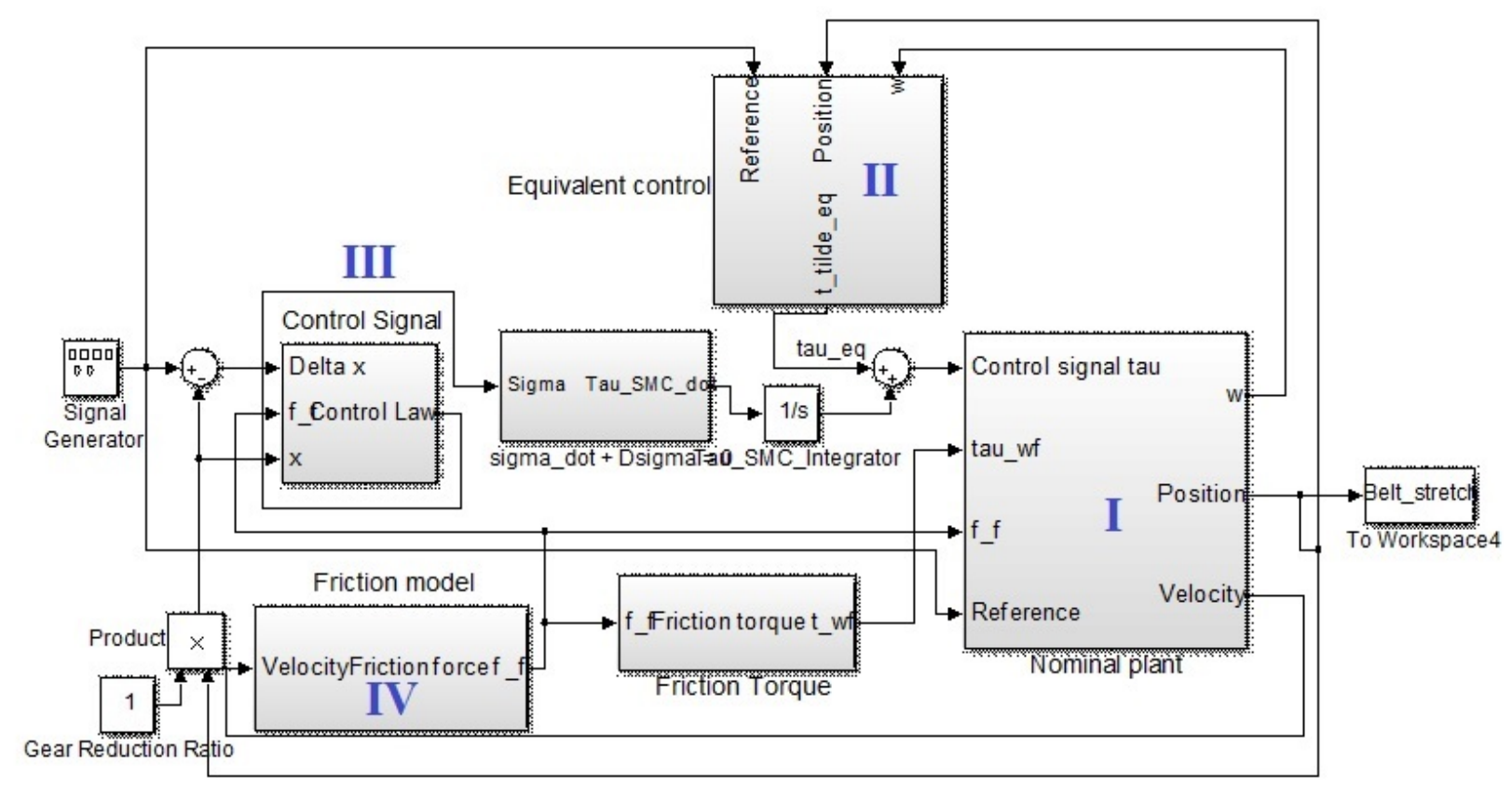

Figure 5: The control and simulation system.

in Figure 6; Equivalent control block for eliminating the chattering (II) in Figure 7; Control creation signal (III): and Friction model (IV) in Figure 8.

In the nominal plant (Figure 5), a noise/disturbance signal with a magnitude of 1 and frequency of $0.5 \mathrm{rad} / \mathrm{sec}$ was added to check the robustness and the chattering free for the control system.

The Belt stretch; Friction torque; and the Control torque to the nominal plant is shown in Figure 9. It can be seen that the Belt stretching block in Figure 8 helps to estimate and minimize the belt stretching to very low value from $3 \mathrm{e}-03 \mathrm{~m}$ and then later it oscillates around the magnitude of 1e-03 $\mathrm{m}$ in correspondence to the varying reference input signal. The control torque, thus, is calculated to minimize the position errors. At the starting point, the torque magnitude is quite high with the approximate magnitude of $985 \mathrm{Nm}$, and later stays within the range of +200 to $-200 \mathrm{Nm}$.

Now, to check the robustness of this system, a phaseship of $\mathrm{pi} / 2$, i.e., $90^{\circ}$ is induced to the reference signal as shown in Figure 10.

In Figure 10, it can be seen that the system is robust enough to deal with the initial offset. It took 1.6 secs to track the reference path and the tracking results are free from chattering.
The system is now tested further with the square tracking. Fiction torque is estimated in the friction mode and used to calculate the friction torque and the control torque. Figure 11 shows the results of belt stretch, friction torque and the control torque for tracking a square reference.

Figure 12 shows the system output performance when it tracks a square reference. Similarly to Figure 10 at the starting point, a high different jump took place because of the initial offset. The output rapidly tracks the reference line. And at time line in $6 \mathrm{~s}$, the reference suddenly is falling and the output follows with a small offset error. But in $13 \mathrm{~s}$ and $19 \mathrm{~s}$, this error is eliminated. The overshooting values are kept at small levels.

Finally, the system is tested tracking on a saw reference with the same magnitude and frequency as the sinuous and square signals. Results in belt stretch, friction torque and the control torque show that the system performs better than previous tracking references. The input torque to keep the belt tracking to the reference is also smaller as shown in Figure 13.

Figure 14 shows the saw tracing performance. At starting point and at $13 \mathrm{~s}$, the tracking overshoots are high due to the big sudden fall of the reference line. However the output very fast tracks to the reference without error.

The above simulations show that the controller has provided chattering-free input in the control torque. The 


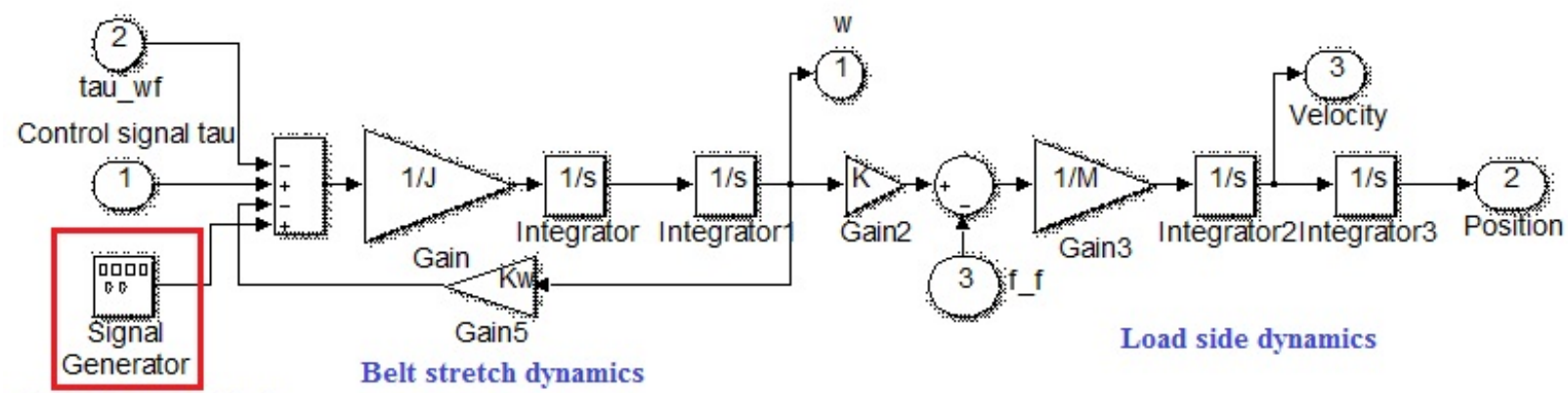

Disturbance added

Figure 6: Nominal plant added with disturbance.
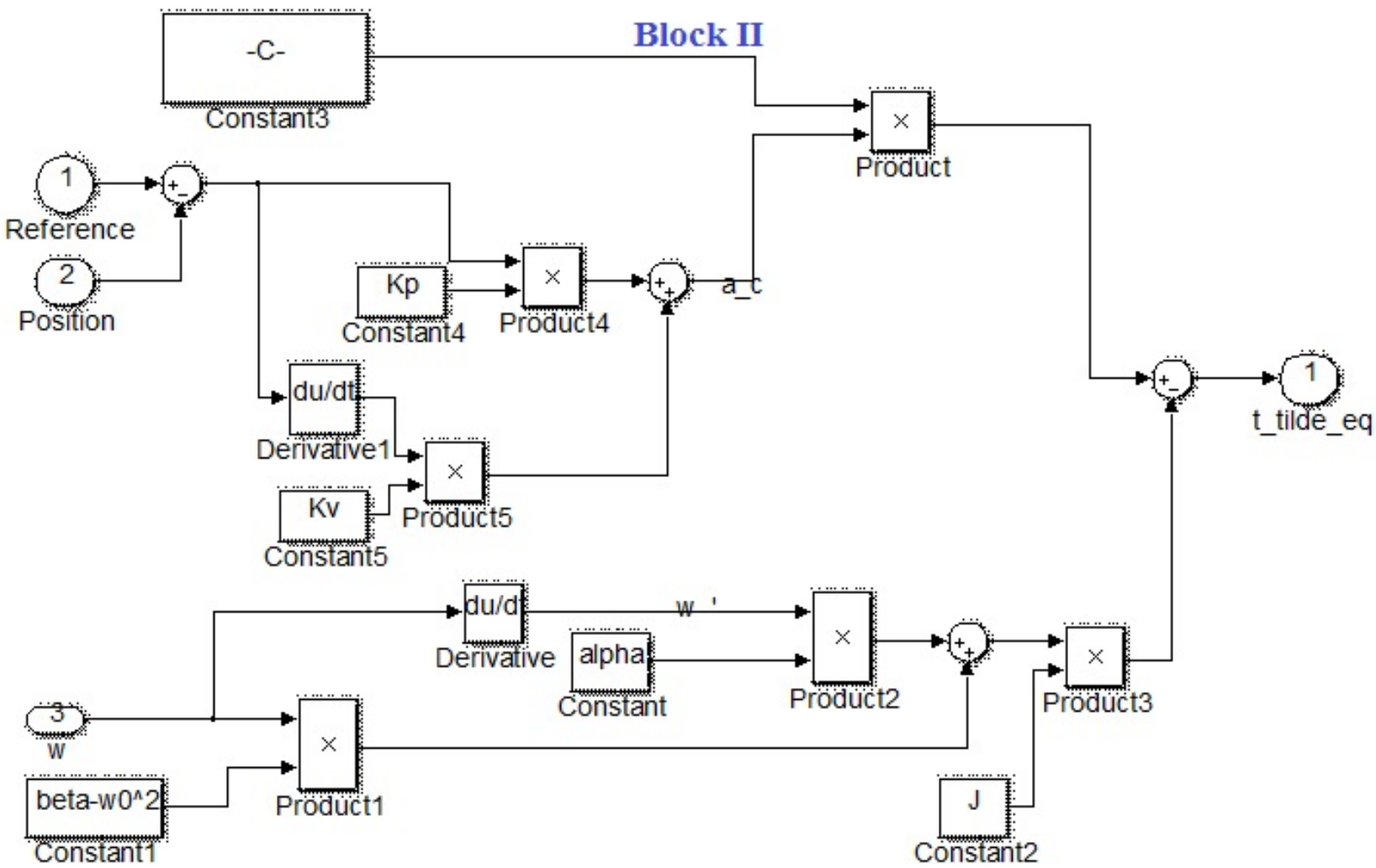

Figure 7: Equivalent control.

tracking errors are small and the system can detect the reference switching and generate the control torque correspondingly. The tracking overshoots become smaller since the system has adapted to those changes.

\section{Conclusion}

This study has included the use of chattering free SMC as in equivalent controller and the belt stretching estimation as in internal friction model into the control law. This SMC provides free chattering and then, the system is safe for all mechanical and electrical devices. The tracking errors are very small. The reference tracking position is achieved at 


\section{Block IV}

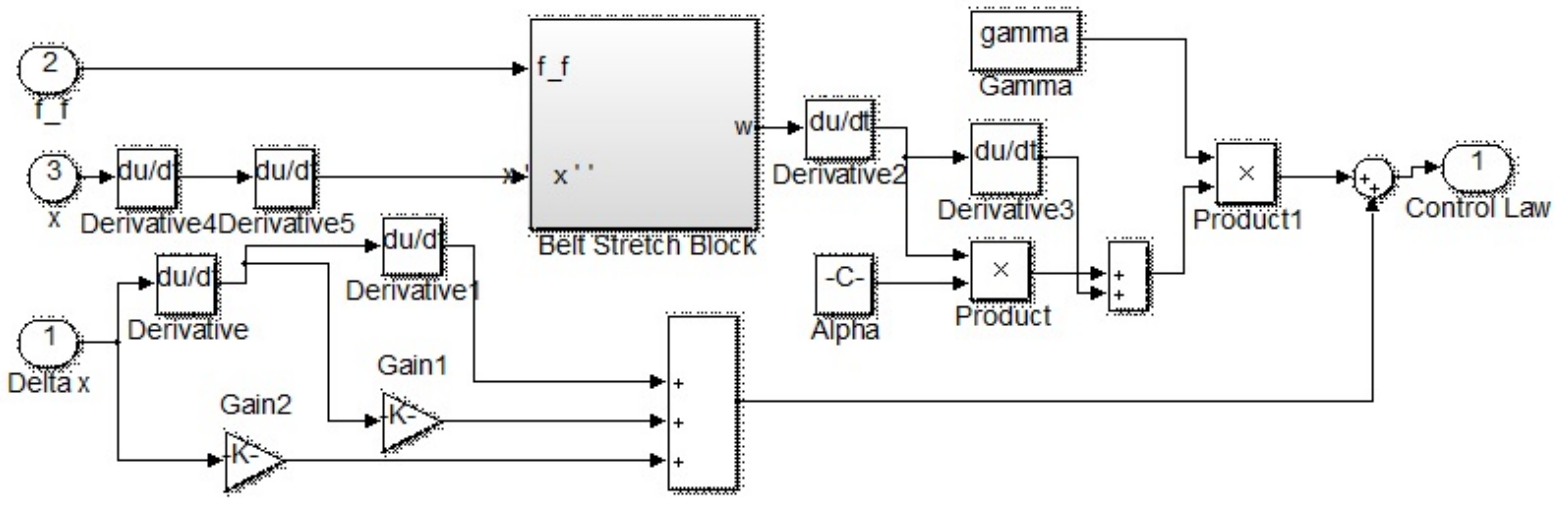

Figure 8: Friction model.
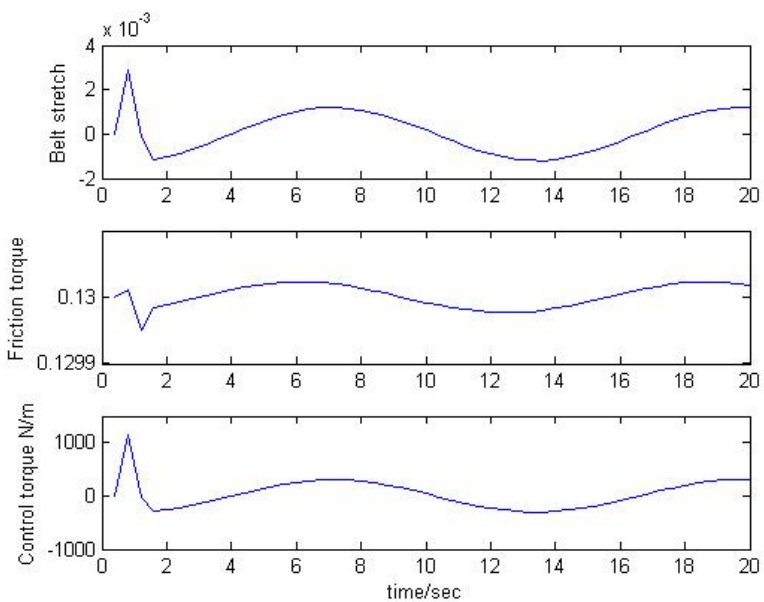

Figure 9: Friction model with sinuous reference.

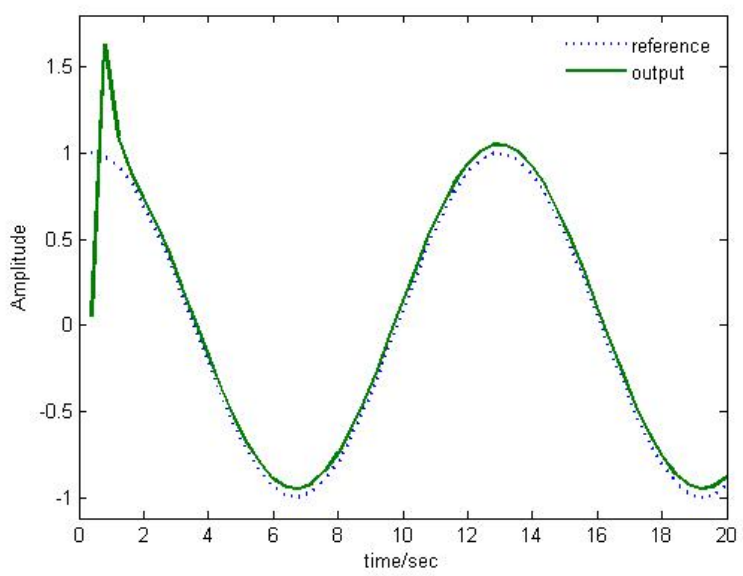

Figure 10: Robust sinuous referenced tracking.
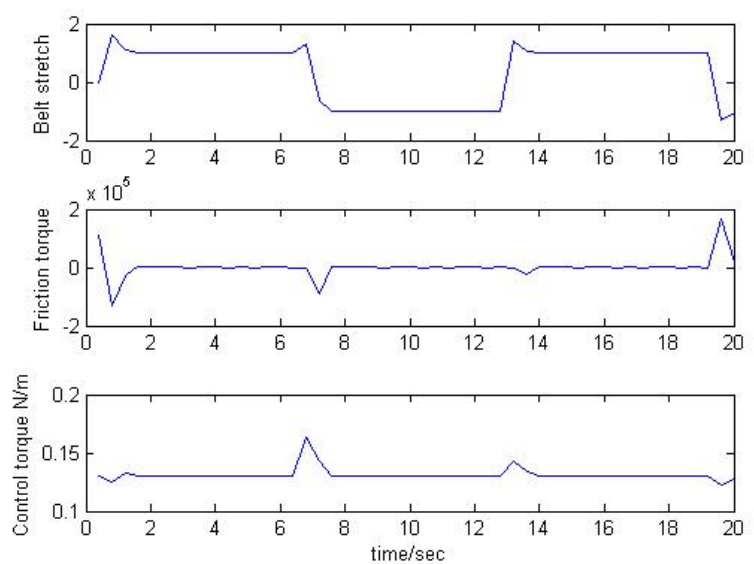

Figure 11: Friction model with square reference.

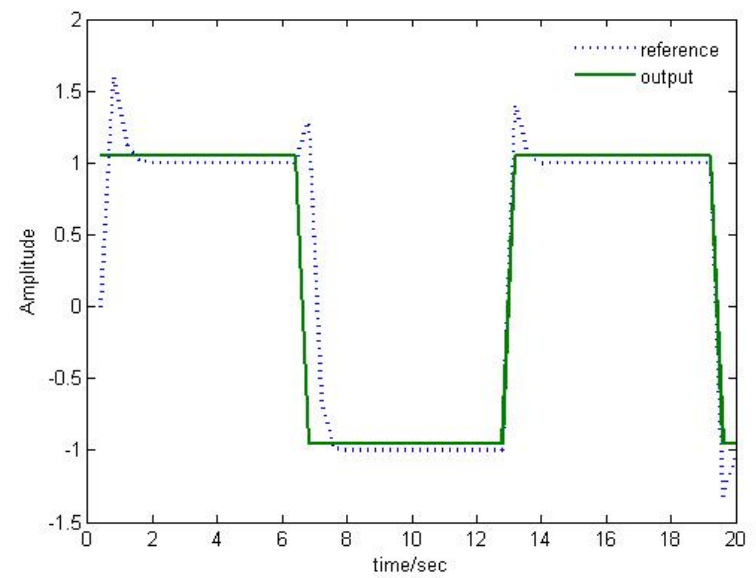

Figure 12: Robust square referenced tracking. 

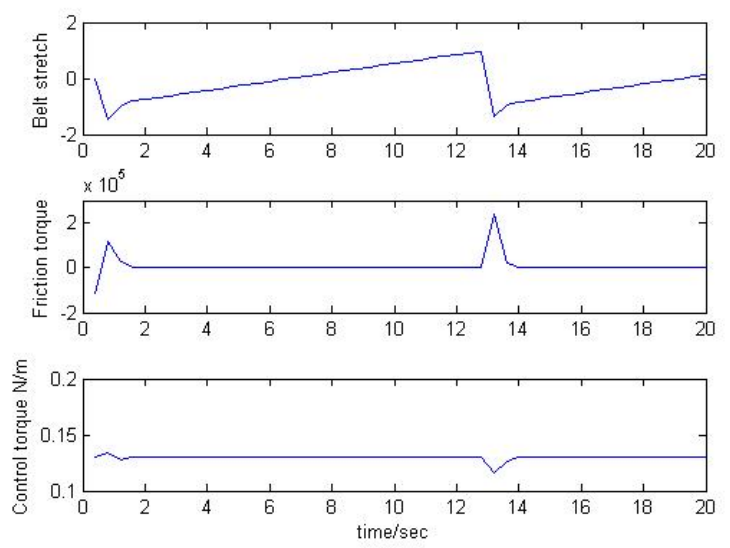

Figure 13: Friction model with saw reference.

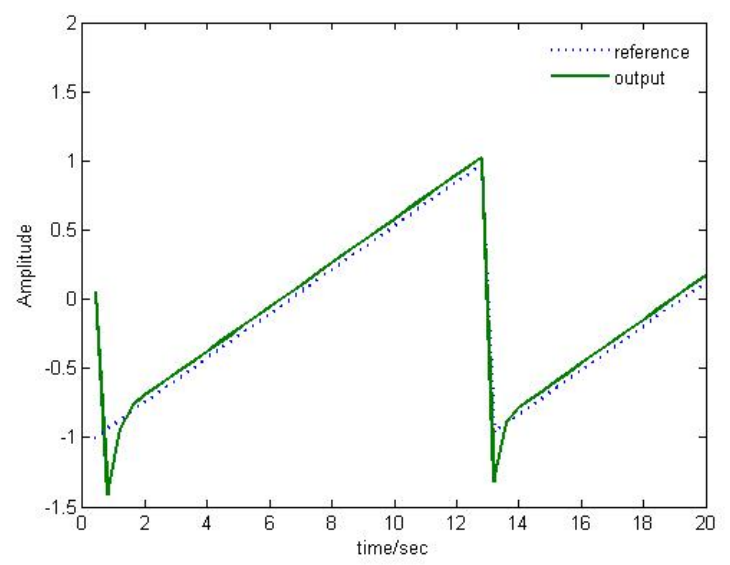

Figure 14: Robust saw referenced tracking.

quite a fast pace and the controller can be able to eliminate any disturbances entering into the system. The magnitude of belt stretching is considerably reduced after the belt stretch control estimator is incorporated into the control law. Experiments are also conducted to test the robustness of the system by changing the initial positions at the starting point. The system swiftly detected and consequently responded to those changes.

In future research, a combination of chattering free SMC and a disturbance observer can be used together to reduce the tracking errors and to deal with the problem of friction as well as disturbance dynamics. Further comparative study of the time delay control in the sliding mode (TDC-SMC) and other control methods can be applied and analyzed to select a better chattering free SMC solution.

Acknowledgement: The authors would like to thank the Department of Electrical Power Engineering and Mecha- tronics, Tallinn University of Technology (TTU) for supporting this research project.

\section{Conflict of interests}

The authors declare that this article is our own research property and there is no conflict of interests regarding the publication of this paper.

\section{References}

[1] Cepon G., Boltezar M., Dynamics of a belt-drive system using a linear complimentarity problem for the belt-pulley contact description, Journal of Sound and Vibration, 2009, 319(3-5), 10191035

[2] Hace A., Jezernik K., Sabanovic A., SMC with disturbance observer for a linear belt drive, IEEE Transactions on Industrial Electronics, 2007, 54(6), 3402-3412

[3] Zhang G., Furusho J., Speed control of two-inertia system by PI/PID control, IEEE Transactions on Industrial Electronics, 2000, 47(3), 603-609

[4] Ellis G., Lorenz R. D., Resonant load control methods for industrial servo drives, In: Proceedings of Annual Meeting of IEEE Industry Applications Society, Rome, Italia, 2000

[5] Levant A., Chattering analysis, IEEE Transactions on Automatic Control, 2010, 55(6), 1380-1389

[6] Sabanovic A., Sozbilir O., Goktug G., Sabanovic N., Sliding mode control of timing-belt servo system, In: Proceedings of IEEE International Conference on Electrical Drives and Power Electronics, Rio de Janeiro, Brazil, 2003

[7] Gurbani C., Kumar V., Designing robust control by sliding mode control technique, AEEE Research Publications, 2013, 3(2), 137144

[8] Chifi-alauhi A., Messaoud H., Chaabane M., Sliding mode control, with integrator, for a class of non-linear systems, Scientific Research Engineering, 2011, 3, 435-444

[9] Lesniewski P., Bartoszewicz A., Hyperbolic tangent based switching reaching law for discrete time sliding mode control of dynamical systems, In: Proceedings of Recent Advances in Sliding Modes, Istanbul, Turkey, 2015

[10] Kochetkov S., Utkin V., Invariance in systems with un-matched perturbations, Journal of Automation and Remote Control, 2013, 74(7), 1097-1127

[11] Shtessel Y., Edwards C., Fridman L., Levant A., Sliding mode control and observation, In: Birkhauser, 2014, ISBN 978-08176-4893-0

[12] Bandyopadhyay B., Thakar V. K., Discrete time output feedback sliding mode control for nonlinear MIMO systems: A step motor case, International Journal of Systems Science, 2008, 39(1), 89104

[13] Zeinali M., Adaptive Chattering-free sliding mode control design using fuzzy model of the system and estimated uncertainties and its application to robot manipulators, In: Proceedings of Recent Advances in Sliding Modes, Istanbul, Turkey, 2015

[14] Zeinali M., Notash L., Adaptive Sliding mode control with uncertainty estimator for robot manipulators, Journal of Mechanism and Machine Theory, 2010, 45(1), 80-90 
[15] Minh V. T., Abdul Rani A. M., Modeling and control of distillation column in a petroleum process, Mathematical Problems in Engineering, Open Access, 2009, art. ID 404702

[16] Wanga B., Shic P., Karimi H., Fuzzy sliding mode control design for a class of disturbed systems, Journal of Franklin Institute, 2014, 351, 3593-3609

[17] Minh V. T., Hashim B., Awang M., Development of a real-time clutch transition strategy for parallel hybrid electric vehicle, Journal of Systems and Control Engineering, 2012, 226(2), 188203
[18] Minh V. T., Afzulpukar N., Muhamad W., Fault detection model based controller for process system, Asian Journal of Control, 2011, 13(3), 382-397

[19] Minh V. T., Pumwa J., Fuzzy logic and slip controller of clutch and vibration for hybrid vehicle, International Journal of Control, Automation and Systems, 2013, 11(3), 526-532 